\title{
Phonon-like Hydrogen-Bond Modes in Protic lonic Liquids
}

\author{
Judith Reichenbach, Stuart A. Ruddell, Mario González-Jiménez, ‘ Julio Lemes, David A. Turton, \\ David J. France, and Klaas Wynne*(0) \\ School of Chemistry, WestCHEM, University of Glasgow, Glasgow G12 8QQ U.K.
}

\section{Supporting Information}

ABSTRACT: Gigahertz- to terahertz-frequency infrared and Raman spectra contain a wealth of information concerning the structure, intermolecular forces, and dynamics of ionic liquids. However, these spectra generally have a large number of contributions ranging from slow diffusional modes to underdamped librations and intramolecular vibrational modes. This makes it difficult to isolate effects such as the role of Coulombic and hydrogenbonding interactions. We have applied far-infrared and ultrafast optical Kerr effect spectroscopies on carefully selected ions with a greater or lesser degree of symmetry in order to isolate spectral signals of interest. This has allowed us to demonstrate the presence of longitudinal and transverse optical phonon modes and a great similarity of alkylammonium-based protic ionic liquids to liquid water. The data show that such phonon modes will be present in all ionic liquids, requiring a reinterpretation of their spectra.

$\mathrm{I}_{\mathrm{oin}}^{\mathrm{o}}$ onic liquids are organic salts with melting points closer to those of traditional solvents than purely inorganic salts. They retain the useful physical properties associated with molten salts, such as low vapor pressure and a wide electrochemical window. This combination makes them candidates for applications in synthesis, catalysis, electrochemistry, and battery development. $^{1-4}$ Protic ionic liquids, which are capable of forming hydrogen bonds, are currently under intense investigation as electrolytes in batteries and hydrogen-based fuel cells. ${ }^{1,3}$ Hydrogen bonding is thought to play a major role in determining the physical properties of ionic liquids in general and protic ionic liquids in particular. ${ }^{5-7}$

Vibrational and relaxational modes associated with hydrogen bonding are expected to occur in the gigahertz to terahertz frequency range. Spectroscopic studies have been carried out on ionic liquids in this frequency domain using far-infrared and terahertz time-domain spectroscopy $(\mathrm{THz}-\mathrm{TDS})^{5-8}$ as well as Raman and optical Kerr effect (OKE) spectroscopy. ${ }^{9-12}$ However, there are many other motions besides those associated with hydrogen bonding that give rise to spectral bands in this range, including librations, low-frequency intramolecular vibrations, and orientational relaxation. Therefore, it is difficult to obtain reliable information on hydrogen bonding.

In previous work on (nonionic) liquids and solutions, we have shown that highly symmetric molecules or even atoms can be used to "switch off" single-molecule-related dynamics in order to isolate intermolecular dynamics. ${ }^{13,14}$ The far-infrared spectrum is proportional to the dipole-dipole correlation function $\langle\mu(0) \mu(t)\rangle$, where $\mu$ is the dipole moment density, while the OKE (or reduced depolarized Raman) spectrum is proportional to the polarizability-polarizability correlation function $\langle\alpha(0) \alpha(t)\rangle$, where $\alpha$ is the collective anisotropic polarizability tensor. ${ }^{15,16}$ In molecules or ions with a center of inversion or tetrahedral symmetry, the molecular dipole moment and anisotropic polarizability tensor are by definition zero, and the spectra are solely determined by intermolecular dynamics. These dynamics should be the same whether measured using OKE or far-IR spectroscopy and should therefore give rise to corresponding bands in the two spectra (although with different relative amplitudes).

Here we will show that this effect can be used in the study of hydrogen bonding in protic ionic liquids. Ions were initially chosen to switch off all of the intramolecular signals through symmetry. The symmetry was then broken gradually in order to switch on particular spectral signals one by one. This allowed us to isolate hydrogen-bonding modes in protic ionic liquids and demonstrate their phonon-like character.

The ionic liquids were studied using OKE and far-infrared Fourier-transform infrared spectroscopies (see the Supporting Information (SI)). OKE experiments were carried out with femtosecond laser pulses using pump-probe delay times of up to $1 \mathrm{~ns}$. The Fourier transform of the time-domain signal results in a depolarized reduced Raman spectrum with a good signalto-noise ratio between $\sim 1 \mathrm{GHz}\left(0.03 \mathrm{~cm}^{-1}\right)$ and $\sim 15 \mathrm{THz}$ $\left(500 \mathrm{~cm}^{-1}\right) \cdot{ }^{17,18}$

Initial OKE and far-IR measurements were carried out on the ionic liquid tetrabutylammonium chloride, which consists of ions that should predominantly show intermolecular dynamics in the spectra on account of their high symmetry (see Figure 1). Torsional modes are expected to be weak in the OKE spectrum and very weak in the far-IR spectrum). In both spectra, one main band is visible below $150 \mathrm{~cm}^{-1}$, with less intense vibrational bands at higher frequency. However, while the main band appears symmetric in the OKE spectrum, it shows an obvious shoulder at lower frequency in the far-IR spectrum. Additionally, the OKE spectrum shows two vibrational bands while the far-IR spectrum shows only one.

The vibrational bands were fitted with Brownian oscillator functions, while the main band in both the OKE and far-IR spectra was fitted with a common set of Gaussians with identical spectral parameters (frequency and damping but not amplitude; see the SI for a more detailed discussion of the

Received: March 27, 2017

Published: May 16, 2017 
(a)

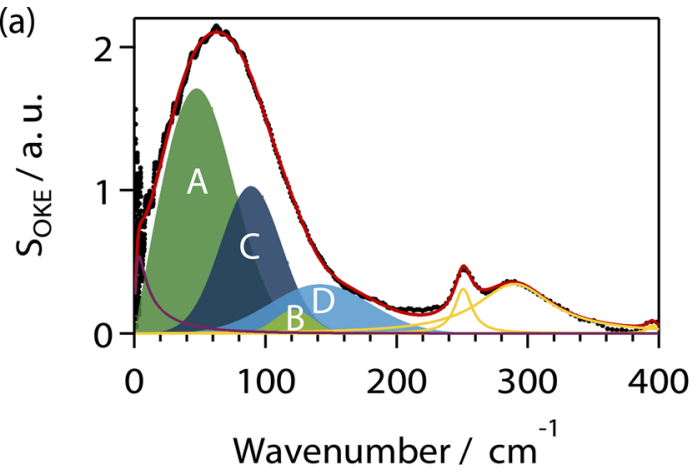

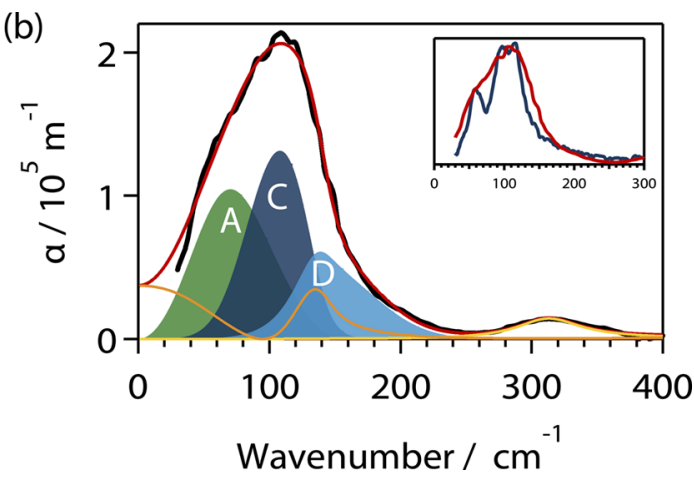

Figure 1. Terahertz spectra and fits for liquid and crystalline tetrabutylammonium chloride. (a) Experimental OKE spectrum of the liquid at $90{ }^{\circ} \mathrm{C}$ (black), the overall fit (red), and component functions of the fit (blue and green). (b) Experimental far-IR spectrum of the liquid at $90^{\circ} \mathrm{C}$ (black), the overall fit (red), the contribution to the absorption due to reflectivity (orange), and component functions of the fit (blue and green). The inset shows a comparison of the far-IR spectra of the liquid (red) and the crystal (dark blue; 2:1 v/v mixture of polyethylene with tetrabutylammonium chloride) at room temperature.
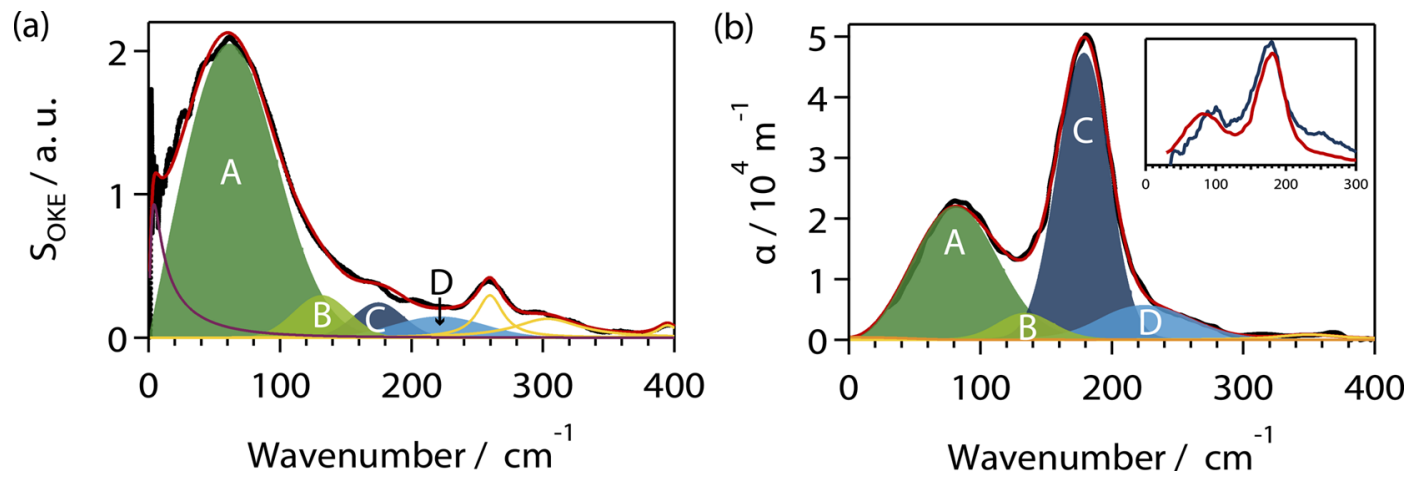

Figure 2. Terahertz-frequency-domain spectra and fits for liquid and crystalline tributylammonium chloride showing a hydrogen-bonding-induced split. Shown are the (a) OKE and (b) far-IR spectra of the liquid at $80{ }^{\circ} \mathrm{C}$ and a comparison with the pellet far-IR spectrum (inset) at room temperature. Color coding as in Figure 1.

fitting procedure). As described in the SI, bands that are common to the far-IR and OKE spectra (such as the band labeled A in Figure 1) typically peak at slightly different frequencies because of the different inherent frequency dependences of the two techniques. ${ }^{16}$ A Debye function was used to account for diffusive modes below $10 \mathrm{~cm}^{-1}$, which were not clearly observable in the far-IR spectra. The main band seen in the far-IR spectrum could be fit with two Gaussian functions of similar intensity, A at $47 \mathrm{~cm}^{-1}$ and C at $89 \mathrm{~cm}^{-1}$ (see Table SI1). A third Gaussian function, D, was needed to fit the tail at higher frequencies in both spectra. The inset of Figure $1 \mathrm{~b}$ shows the far-IR spectrum of crystalline tetrabutylammonium chloride mixed with polyethylene, which shows the two bands $\mathrm{A}$ and $\mathrm{C}$ as narrow and clearly separated peaks, confirming the validity of the fitting procedure used for the liquid.

Next the symmetry of the cation was reduced and hydrogen bonding was introduced by replacing tetrabutylammonium with tributylammonium. The OKE and far-IR spectra of liquid tributylammonium chloride are shown in Figure 2. Comparison of Figure $2 \mathrm{~b}$ with Figure $1 \mathrm{~b}$ shows that the single band is now split into two in tributylammonium chloride. The split between the $\mathrm{A}$ and $\mathrm{C}$ bands is $114 \mathrm{~cm}^{-1}$ in tributylammonium chloride (compared with $42 \mathrm{~cm}^{-1}$ in tetrabutylammonium chloride) and persists in the crystalline spectrum (see the inset of Figure $2 b$ ).

In order to switch off hydrogen bonding but maintain asymmetry in the cation, tributylmethylammonium chloride was also studied. As can be seen in Figure 3a, the spectrum of liquid tributylmethylammonium chloride is similar to that of tetrabutylammonium chloride in showing no split in the far-IR spectrum. Therefore, this split is not associated with the break in symmetry but rather with the formation of hydrogen bonds. Figure $3 \mathrm{~b}$ compares the far-IR spectra of liquid tributylammonium chloride and bromide, which leads to a shift of both the $\mathrm{A}$ and $\mathrm{C}$ bands toward lower frequency. The average ratio of the center frequencies is 0.64 for the tributylammonium halides. For the tetrabutylammonium halides (Figure SIla) and the tributylmethylammonium halides (Figure SI1b) an average ratio of 0.70 was observed.

Fitting the OKE and far-IR data shows that a small number of inhomogeneously broadened underdamped bands appear in the $0-200 \mathrm{~cm}^{-1}(0-7 \mathrm{THz})$ range. Underdamped bands in the terahertz range are usually associated with librational modes of the molecules in the liquid. However, for such librational bands to appear in the OKE spectrum, the molecular polarizability tensor must be anisotropic. Similarly, for them to appear in the far-IR spectrum, the molecules must have a dipole moment. Because of its tetrahedral symmetry, tetrabutylammonium has neither. Of course, the tetrahedral symmetry of tetrabutylammonium is likely to be distorted in the liquid, and therefore, one may expect a weak OKE signal and weak far-IR absorption. However, as can be seen in Figure $1 b$, the peak absorption is $2.2 \times 10^{5} \mathrm{~m}^{-1}$, which is very strong and comparable to that of liquid water. ${ }^{19}$ Similarly, one might expect torsional modes in this region, but these are also predicted to be weak. This strongly suggests that the absorption is caused by the motion of the relatively light and mobile chloride anion in a cage of 

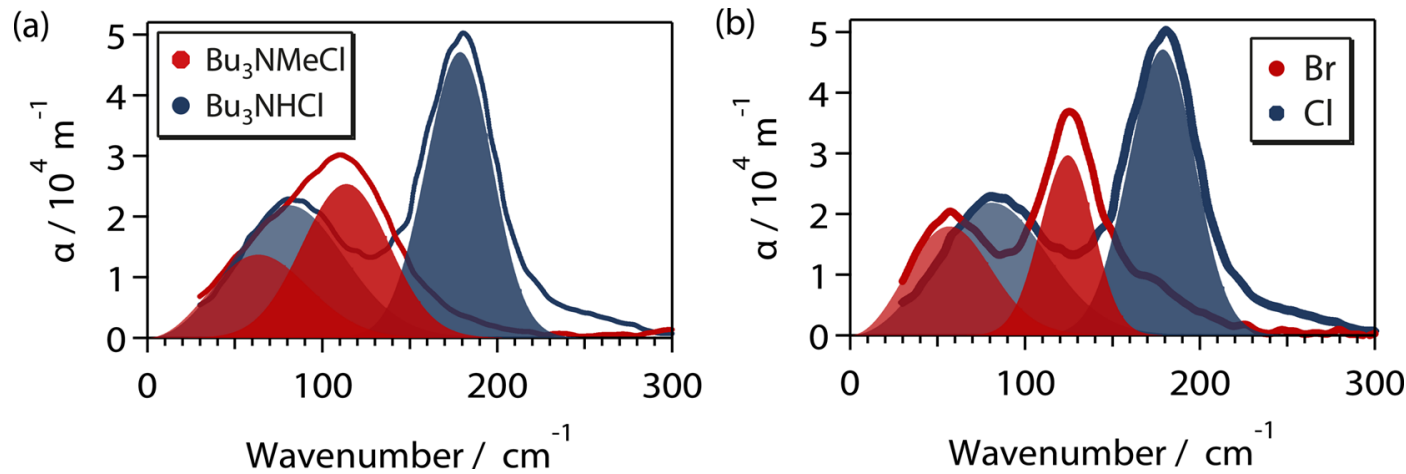

Figure 3. Effect of changing the cation or the anion on the terahertz-frequency-domain far-IR spectra. (a) Comparison of the far-IR spectra of liquid tributylammonium and tributylmethylammonium chloride. (b) Comparison of the far-IR spectra of liquid tributylammonium chloride and bromide.

tetrabutylammonium cations. ${ }^{20}$ Such a motion corresponds to a lattice phonon mode, and momentum conservation would dictate that this should be the longitudinal optical (LO) or transverse optical (TO) phonon near the zone center $(k=0)$. The Figure $1 \mathrm{~b}$ inset shows that the broad band in the liquid narrows to two bands in the solid, consistent with the assignment as the LO and TO phonons.

Changing the anion from chloride to bromide in the alkylammonium salts gives rise to a change in the center frequency of phonon bands. The frequency change for two different salts 1 and 2 due to a change in masses only can be calculated using $\omega_{1} / \omega_{2}=\sqrt{m_{\text {anion, } 1}^{-1}+m_{\text {cation, } 1}^{-1}} /$ $\sqrt{m_{\text {anion,2 }}^{-1}+m_{\text {cation,2 }}^{-1}}$ at the zone center $(k=0)$ and $\omega_{1} / \omega_{2}=$ $\sqrt{m_{\mathrm{x}, 2} / m_{\mathrm{x}, 1}}$ at the zone edge $(k=2 \pi)$, where $m_{\mathrm{x}}$ is the mass of the lighter ion (the anion in this case). ${ }^{21}$ The average ratio calculated from our fits is 0.64 for the protic ionic liquids (tributylammonium chloride and bromide; 0.62 for the A band and 0.69 for the $\mathrm{C}$ band) and 0.70 for the nonprotic ionic liquids (tetrabutylammonium chloride/bromide and tributylmethylammonium chloride/bromide). The expected shift is 0.67 at the zone edge and 0.72 to 0.73 at the zone center. Thus, the experimental values are closer to those predicted for the zone edge, which can be understood as a localization effect caused by the disorder in the liquid that reduces the phonon dispersion.

It is immediately obvious from a comparison of Figure $1 \mathrm{~b}$ with Figure $2 \mathrm{~b}$ and Figure 3 that the peak absorption is an order of magnitude higher in tetrabutylammonium chloride than in the other ionic liquids. The absorption is a frequencydependent quantity (see eq SI6), but the amplitudes used in fitting and listed in Table SI1 are not frequency-dependent and scale with the square of the (transition) dipole moment. ${ }^{16}$ By comparison of the amplitudes of band $\mathrm{A}$, which is the band with the highest amplitude, it can be seen that the ratio is $>9$ for the chlorides and $>18$ for the bromides. This shows that the anions are less mobile in the tributylammonium and tributylmethylammonium ionic liquids. The simplest explanation is that the anions and cations are more strongly paired in these liquids because of the easier accessibility of the positive charge centered approximately on the nitrogen atom and therefore greater Coulombic interaction.

In the tributylammonium salts there is an additional splitting of the LO and TO phonons due to additional hydrogenbonding interactions. It is generally expected that the LO phonon has a higher frequency than the TO phonon. ${ }^{22}$ Hence, the A band is identified as the TO (hydrogen-bond bend) and the $\mathrm{C}$ band as the LO (hydrogen-bond stretch) phonon band. This behavior is similar to that seen in the terahertz spectrum of liquid water, which exhibits $\sim 2 \mathrm{THz} \mathrm{TO}$ and $\sim 5 \mathrm{THz} \mathrm{LO}$ phonon modes at the zone edge. ${ }^{23}$ As can be seen in Figure 2, there is a large drop in intensity of the LO band relative to the TO band on going from the far-IR spectrum to the OKE spectrum, which is consistent with other examples in the literature. $^{24,25}$

The band labeled A has previously been assigned to librational motions of the cation combined with a weak contribution of unspecific cation-anion interaction modes. ${ }^{5,26}$ Of course, the spectra of ionic liquids with less symmetric cations could have contributions from cation librations. The calculated permanent dipole moments of tetraethylammonium, triethylmethylammonium, and triethylammonium ions are 0.7 , 1, and 2.4-3.4 D (depending on the isomer; see the SI), respectively. Thus, even though the cations have small dipole moments relative to their volumes, the far-IR absorption is very strong and strongest for the cation with the smallest dipole moment, ruling out an origin of the far-IR absorption in librational motion. Similarly, the calculated molecular polarizability tensors for the three cations are very nearly isotropic (see the SI). As the OKE signal strength is large in all of the ionic liquids studied here, this again rules out an origin of the OKE spectra in librational motion. Hence, the data show that by far the major contribution in this spectral range comes from translational motion of the anion relative to the cations, which we have identified as the LO and TO phonons.

It is found that the OKE signal strengths for all of the alkylammonium ionic liquids studied here are approximately the same and only a factor of 2-3 weaker than those of typical pyridinium- and imidazolium-based ionic liquids. The far-IR absorption strengths in the few-terahertz region are also similar for alkylammonium- ${ }^{24}$ and imidazolium-based ${ }^{10}$ ionic liquids. This then implies that TO and LO bands are likely to be significant in the terahertz far-IR and OKE spectra of all ionic liquids irrespective of whether they have significant hydrogen bonding between ions or not. The masses of typical anions used in ionic liquids (e.g., $\mathrm{BF}_{4}^{-}$, dicyanamide, methylsulfonate) are all rather similar to those of chloride and bromide, and therefore, one expects TO and LO bands at terahertz frequencies in the spectra of all ionic liquids. In ionic liquids with less symmetric and strongly anisotropically polarizable cations such as pyridinium and imidazolium, it will be more difficult to observe the TO and LO bands, but their contribution is predicted to be significant. This will require a 
reinterpretation of ionic liquid spectra and will provide new insights into the structure and interactions of these liquids.

\section{ASSOCIATED CONTENT}

\section{S Supporting Information}

The Supporting Information is available free of charge on the ACS Publications website at DOI: 10.1021/jacs.7b03036.

Materials, experimental details, additional data, data analysis, curve-fitting procedures, fit parameters, calculation of dipole moments and molecular polarizabilities, melting points, and references (PDF)

\section{AUTHOR INFORMATION}

\section{Corresponding Author}

*klaas.wynne@glasgow.ac.uk

\section{ORCID}

Mario González-Jiménez: 0000-0002-8853-0588

Klaas Wynne: 0000-0002-5305-5940

\section{Notes}

The authors declare no competing financial interest.

The FTIR and OKE data that support the findings of this study are available in Enlighten: Research Data Repository (University of Glasgow) with the identifier http://dx.doi.org/10. 5525/gla.researchdata.404.

\section{ACKNOWLEDGMENTS}

We thank the Engineering and Physical Sciences Research Council (EPSRC) for support through grants EP/J009733/1, $\mathrm{EP} / \mathrm{J} 00975 \mathrm{X} / 1, \mathrm{EP} / \mathrm{K} 034995 / 1$, and EP/N508792/1.

\section{REFERENCES}

(1) MacFarlane, D. R.; Tachikawa, N.; Forsyth, M.; Pringle, J. M.; Howlett, P. C.; Elliott, G. D.; Davis, J. H.; Watanabe, M.; Simon, P.; Angell, C. A. Energy Environ. Sci. 2014, 7 (1), 232-250.

(2) Armand, M.; Endres, F.; MacFarlane, D. R.; Ohno, H.; Scrosati, B. Nat. Mater. 2009, 8 (8), 621-629.

(3) Smiglak, M.; Pringle, J. M.; Lu, X.; Han, L.; Zhang, S.; Gao, H.; MacFarlane, D. R.; Rogers, R. D. Chem. Commun. 2014, 50, 92289250.

(4) Conrad Zhang, Z. Adv. Catal. 2006, 49, 153-237.

(5) Fumino, K.; Fossog, V.; Wittler, K.; Hempelmann, R; Ludwig, R. Angew. Chem., Int. Ed. 2013, 52 (8), 2368-2368.

(6) Fumino, K.; Reichert, E.; Wittler, K.; Hempelmann, R.; Ludwig, R. Angew. Chem., Int. Ed. 2012, 51 (25), 6236-6236.

(7) Fumino, K.; Peppel, T.; Geppert-Rybczyńska, M.; Zaitsau, D. H.; Lehmann, J. K.; Verevkin, S. P.; Koeckerling, M.; Ludwig, R. Phys. Chem. Chem. Phys. 2011, 13 (31), 14064-14075.

(8) Yamada, T.; Tominari, Y.; Tanaka, S.; Mizuno, M. J. Phys. Chem. B 2015, 119 (51), 15696-15705.

(9) Shirota, H.; Kakinuma, S. J. Phys. Chem. B 2015, 119 (30), 98359846.

(10) Turton, D. A.; Hunger, J.; Stoppa, A.; Hefter, G.; Thoman, A.; Walther, M.; Buchner, R.; Wynne, K. J. Am. Chem. Soc. 2009, 131 (31), 11140-11140.

(11) Castner, E. W.; Wishart, J. F. J. Chem. Phys. 2010, 132 (12), 120901-120901.

(12) Xiao, D.; Rajian, J. R.; Cady, A.; Li, S.; Bartsch, R. a.; Quitevis, E. L. J. Phys. Chem. B 2007, 111 (18), 4669-4677.

(13) Turton, D. A.; Wynne, K. J. Chem. Phys. 2009, 131 (20), 201101-201101.

(14) Turton, D. A.; Hunger, J.; Hefter, G.; Buchner, R.; Wynne, K. J. Chem. Phys. 2008, 128 (16), 161102-161102.

(15) Fecko, C.; Eaves, J.; Tokmakoff, A. J. Chem. Phys. 2002, 117 (3), $1139-1154$
(16) Giraud, G.; Wynne, K. J. Chem. Phys. 2003, 119 (22), 1175311764.

(17) González-Jiménez, M.; Ramakrishnan, G.; Harwood, T.; Lapthorn, A.; Kelly, S.; Ellis, E.; Wynne, K. Nat. Commun. 2016, 7, 11799-11799.

(18) Turton, D. A.; Senn, H. M.; Harwood, T.; Lapthorn, A. J.; Ellis, E. M.; Wynne, K. Nat. Commun. 2014, 5, 3999.

(19) Rønne, C.; Åstrand, P.-O.; Keiding, S. Phys. Rev. Lett. 1999, 82 (14), 2888-2891.

(20) Funkner, S.; Niehues, G.; Schmidt, D. A.; Heyden, M.; Schwaab, G.; Callahan, K. M.; Tobias, D. J.; Havenith, M. J. Am. Chem. Soc. 2012, 134 (2), 1030-1035.

(21) Kittel, C. Introduction to Solid State Physics, 8th ed.; John Wiley \& Sons: New York, 2005.

(22) Srivastava, G. P. The Physics of Phonons; Hilger: Bristol, U.K., 1990.

(23) Walrafen, G. E. J. Phys. Chem. 1990, 94 (6), 2237-2239.

(24) Sonnleitner, T.; Turton, D. A.; Hefter, G.; Ortner, A.; Waselikowski, S.; Walther, M.; Wynne, K.; Buchner, R. J. Phys. Chem. B 2015, 119 (29), 8826-8841.

(25) Fumino, K.; Wulf, A.; Ludwig, R. Angew. Chem., Int. Ed. 2009, 48 (17), 3184-6.

(26) Zaitsau, D. H.; Emel'yanenko, V. N.; Stange, P.; Schick, C.; Verevkin, S. P.; Ludwig, R. Angew. Chem., Int. Ed. 2016, 55 (38), 11682-11686. 